\title{
On the Performance of Coherent and Noncoherent Multiuser Detectors for Mobile Radio CDMA Channels
}

\author{
Hsin-Yu Wu and Alexandra Duel-Hallen \\ Department of Electrical and Computer Engineering \\ Box 7911, North Carolina State University \\ Raleigh, NC 27695 \\ internet: hwu@eos.ncsu.edu, sasha@eos.ncsu.edu
}

\begin{abstract}
We investigate the performance of several suboptimal multiuser detectors for rapidly time varying mobile radio channels. A modified Jakes model is used to simulate a realistic mobile fading channel. The use of the Kalman filter for this channel model is examined. We also analyze the performance of several noncoherent multiuser detectors. Analysis and simulation results indicate that the decorrelator is more robust than other considered multiuser detectors.
\end{abstract}

\section{Introduction}

Multiuser detection has the potential to reduce the Multi-Access Interference (MAI) and solve the near-far problem in the reverse link of a Code Division Multiple Access (CDMA) channel. Several suboptimal multiuser detection schemes with reasonable complexity have been studied. Among these schemes are the linear decorrelator [1], the multistage detector [2], the decision-feedback detector (DF) [3], the successive interference cancellation (SIC) [4], and parallel interference cancellation scheme (PIC) $[4,5]$.

Most evaluations of these multiuser detectors are performed under the ideal assumption of perfect channel estimation. This assumption is not valid in practice. The imperfect channel estimation degrades the performance of multiuser detectors since many multiuser detectors require channel estimates to cancel the MAI and/or to perform coherent reception. The main purpose of this paper is to compare robustness of these detectors with the Kalman channel estimators for realistic channels.

Using the Kalman filter to estimate fading channel coefficients was first suggested in [6], and more work can be found in [7]. Most of this work modeled the fading channel as an auto-regressive (AR) process in order to apply the Kalman filter. In our work, we use a more realistic channel model-Jakes model [8] with modification of [9]. Combinations of Kalman filters and multiuser detectors were also studied previously. In [10], it was shown that a multiuser detector can be decoupled from a channel estimator, and [11] showed that the Kalman filter can be configured to estimate all the users' channel coefficient jointly or disjointedly. We adopt the disjoint estimation in our work. With the AR channel model, the Bit Error Rate (BER) analysis of the decorrelator, the DF, and the two-stage detector (2S) with the disjoint Kalman channel estimators can be found in $[12,13,19,21]$.

This paper differs from our previous work in the following aspects. (1) We use a more realistic channel model-the modified Jakes model, instead of a second order AR process to generate channel coefficients. (2) We test the use of Kalman filter to estimate a fading process which does not exactly obey the Gauss-Markov model. The signal model embedded in the Kalman filter is a second order AR process. (3) In the performance evaluation, in addition to previously studied multiuser detectors with Kalman channel estimator (the decorrelator, the $2 \mathrm{~S}$ and the $\mathrm{DF}$ ), we include two simpler interference cancellation schemes--the SIC and the PIC. These two schemes are implemented noncoherently and do not require channel estimation.

The paper is organized as follows: Section II discusses the approximation of the modified Jakes model by an AR process which is used as a signal model of a Kalman filter. Section III briefly reviews the multiuser detectors to be compared. Section IV addresses the BERs of these multiuser detectors. Section V presents the simulation results and the performance comparison of the multiuser detectors. Finally, the conclusions and the future work are contained in Section VI.

\section{Channel model and Kalman filter}

A fading channel can be modeled by a popular Jakes model [8]. In [9], a modified Jakes model was introduced. The autocorrelation function of the modified Jakes model approximates that of a Rayleigh fading channel,

$$
\Phi(\tau)=E\left\{C(t) C^{*}(t+\tau)\right\}=J_{0}\left(2 \pi f_{d} \tau\right)
$$

where $\mathrm{J}_{0}$ is the Bessel function of the zero-th kind, $\mathrm{C}(\mathrm{t})$ is the fading waveform, and $f_{d}$ is the maximum Doppler shift. In this paper, the discrete time channel coefficient is denoted by $\mathrm{C}(\mathrm{k})=\mathrm{C}(\mathrm{kT})$, where $\mathrm{T}$ is the sampling period. The variance of the channel coefficient is $\mathrm{E}\left\{|\mathrm{C}(\mathrm{k})|^{2}\right\}=1$.

It is well known that utilization of the Kalman filter for estimation of a process requires the knowledge of the statistical nature of that process. This statistical information leads to the construction of a Gauss-Markov signal model which is embedded in the implementation of the Kalman filter. Therefore, to use the Kalman filter for fading channel estimation, we need to define the signal model for a fading process generated by the modified Jakes model. One way to define a signal model for a fading channel is to approximate the power spectral density of a fading channel using a high order low pass filter. For example, an eighth-order elliptic filter was used in [14]. This high order approximation will result in the increase of the dimension of the Kalman filter and is not desirable in practical implementation. It is possible to approximate the modified Jakes model by a lower order AR process, such as a second order AR process [15]. In [16], Lindbom observed that in choosing the parameters of the AR signal model for a realistic fading channel, the Doppler frequency used in the AR signal model should be compensated by a factor of $\sqrt{2}$ from the real Doppler frequency.

The factor of $\sqrt{2}$ compensation can be shown more obviously by observing the autocorrelation functions of a Rayleigh fading channel and a second order AR process [17]. Let $C^{\prime}(\mathrm{k})$ be a discrete-time sequence. This sequence is generated by a second order AR process that the Kalman filter 
uses to model the actual channel coefficient $\mathrm{C}(\mathrm{k})$, and can be expressed by

$$
C^{\prime}(k)=-a_{1} C^{\prime}(k-1)-a_{2} C^{\prime}(k-2)+w(k),
$$

where $\mathrm{w}(\mathrm{k})$ is the driving noise of the process given by a zeromean complex white Gaussian process. The AR process parameters $\mathrm{a}_{1}$ and $\mathrm{a}_{2}$ are determined by the locations of the poles of the transfer function of the AR process; they are defined by

$$
a_{1}=-2 r_{d} \cos \left(2 \pi f_{d}^{\prime} T\right) \quad a_{2}=r_{d}^{2},
$$

where $2 \pi f_{d}^{\prime}$ and $\mathrm{r}_{\mathrm{d}}$ specify the angle and the pole radius inside the unit circle on a complex plane. The radius is chosen very close to one to model the spectral peaks at the maximum Doppler frequency of the fading process. Let $\Phi^{\prime}(m)=E\left\{C^{\prime}(k) C^{\prime *}(k-m)\right\}$ be the autocorrelation function of the second order AR process. Since the variance of the actual channel coefficient is one, we define $\Phi^{\prime}(0)=1$. From (2), $\Phi^{\prime}(1)=-a_{1} /\left(1+a_{2}\right)$, and the values of $\Phi^{\prime}(m)$ for $m \geq 2$ can be obtained by solving the difference equation,

$$
\Phi^{\prime}(m)=-a_{1} \Phi^{\prime}(m-1)-a_{2} \Phi^{\prime}(m-2), \mathrm{m} \geq 2 .
$$

The solution to (4) can be approximated by

$$
\Phi^{\prime}(m) \approx r_{d}^{m-2} \cos \left(2 \pi f_{d}^{\prime} T(m-2)\right), \mathrm{m} \geq 2 .
$$

From (1), the autocorrelation function $\Phi(m)$ of a fading coefficient $\mathrm{C}(\mathrm{k})$ is

$$
\Phi(m)=E\left\{C(k) C^{*}(k-m)\right\}=J_{0}\left(m 2 \pi f_{d} T\right) .
$$

Using Taylor series expansion for (5) and (6), we have

$$
\begin{gathered}
\Phi(m)=1-\frac{\left(2 \pi f_{d} T m\right)^{2}}{2^{2}}+o\left(\left[2 \pi f_{d} T m\right]^{2}\right) . \\
\Phi^{\prime}(m)=1-\frac{\left(2 \pi f_{d}^{\prime} T r_{d}(m-2)\right)^{2}}{2 !}+o\left(\left[2 \pi f_{d}^{\prime} T r_{d}(m-2)\right]^{2}\right) .
\end{gathered}
$$

To make $\Phi^{\prime}(m) \approx \Phi(m)$ for moderate vaues of $\mathrm{m}$, we compare only the first two expansion terms since the terms $2 \pi f_{d} T m$

and $2 \pi f_{d}^{\prime} T r_{d}(m-2)$ are small. We have

$$
f_{d}^{\prime} \approx \frac{1}{\sqrt{2}} f_{d} .
$$

The approximation in the last term in (9) is achieved when $r_{d}$ approaches 1 . This result indicates that a second order AR process with the parameter $\mathrm{fd}^{\prime}$ is approximately equivalent to a

fading process with maximum Doppler shift $\sqrt{2} \mathrm{fd}$ '. Figure 1 shows the autocorrelation functions of a fading process and the second order AR process with and without the factor of $\sqrt{2}$ compensation.

With this adjustment of the parameters of the signal model embedded in the Kalman filter, we can implement the Kalman filter as follows. With the state vector $\mathbf{X}(k)=\left[C^{\prime}(k) C^{\prime}(k-1)\right]^{T}$, the signal model (2) can be rewritten by

$$
\mathbf{X}(k+1)=\mathbf{A}(k) \mathbf{X}(k)+\mathbf{G}(k) w(k),
$$

where $\mathbf{A}=\left[\begin{array}{cc}-a_{1} & -a_{2} \\ 1 & 0\end{array}\right]$ and $\mathbf{G}=\left[\begin{array}{ll}1 & 0\end{array}\right]^{T}$. The observation process considered here is a discrete-time received signal distorted by a multiplicative fading coefficient and an additive white Gaussian noise,

$$
z(k)=\sqrt{E b} C^{\prime}(k) b(k)+v(k),
$$

where $\mathrm{E}_{\mathrm{b}}, \mathrm{b}(\mathrm{k})$ and $\mathrm{v}(\mathrm{k})$ are the symbol energy, the information bit, and the additive noise. Note that the fading coefficient $\mathrm{C}^{\prime}(\mathrm{k})$ should be replaced by the fading process $\mathrm{C}(\mathrm{k})$ in actual simulation. The received signal is then given by

$$
z(k)=\mathbf{H}^{T}(k) \mathbf{X}(k)+v(k),
$$

where $\mathbf{H}(k)=\sqrt{E b} \quad b(k)\left[\begin{array}{ll}1 & 0\end{array}\right]^{T}$. The operation of this

Kalman filter for the AR channel model in the decision-directed mode is described in $[13,17,19]$.

In summary, we generate the channel coefficients by the modified Jakes model and use the Kalman filter with embedded the second order AR signal model. We find that the behavior of the estimator is similar to the case when channel coefficients are generated by the AR model [13]. In particular, the reversal phenomenon [19] caused by deep fades, where the channel estimates have nearly 180 degree phase shift relative to the channel coefficients, still occurs. Therefore, as proposed in [13], we encode data differentially to avoid long error bursts associated with the reversal phenomenon.

\section{Multiuser detectors}

In this section, we briefly describe the multiuser detectors used in our performance evaluation. Consider a DS/CDMA system with $\mathrm{N}$ users. The received signal is fed into a matched filter bank, where the filters match signature waveforms of all users. The k-th sampled output of the matched filter bank can be written by a column vector $\mathbf{y}(\mathrm{k})$ with the dimension $\mathrm{N}$ :

$$
\mathbf{y}(k)=\mathbf{R W}(k) \mathbf{b}(k)+\mathbf{n}(k),
$$

where $\mathbf{R}$ is the normalized cross-correlation matrix of signature waveforms with unity diagonal entries. The column vector $\mathbf{b}(\mathrm{k})$ consists of the information bits of $\mathrm{N}$ users. The diagonal channel gain matrix $\mathbf{W}(\mathrm{k})$ has components $\mathbf{W}_{\mathrm{n}, \mathrm{n}}=\sqrt{E b_{n}} \mathrm{C}_{\mathrm{n}}(\mathrm{k})$ where $E b_{n}$ is the bit energy and $C_{n}(k)$ is the fading channel coefficient. For Rayleigh fading channels, the channel coefficient is a zero mean, unity variance complex Gaussian random variable. The colored noise vector $\mathbf{n}(\mathrm{k})$ has covariance matrix $\mathrm{E}\left(\mathbf{n}(\mathrm{k}) \mathbf{n}^{\mathrm{H}}(\mathrm{k})\right)=\mathbf{R} \mathrm{N}_{0}$ where $\mathbf{n}^{\mathrm{H}}(\mathrm{k})$ denotes conjugate transpose of $\mathbf{n}(\mathrm{k})$.

The conventional detector makes a decision for user $n$ by using the output of the matched filter directly, i.e., $b_{n}(k)=\operatorname{sgn}\left\{\operatorname{Re}\left[y_{n}(k) C_{n}^{*}(k)\right]\right\}$, where $C_{n}(k)$ is the channel estimate of user $n$. The performance of the conventional detector is poor due to MAI. The decorrelator [1] is obtained by multiplying the outputs of the matched filter bank $\mathbf{y}(\mathrm{k})$ in (13) by the inverse of the correlation matrix $\mathbf{R}$ ( $\mathbf{R}$ is assumed to be nonsingular):

$$
\mathbf{z}(k)=\mathbf{R}^{-1} \mathbf{y}(k)=\mathbf{W}(k) \mathbf{b}(k)+\mathbf{v}(k),
$$

where the noise vector $\mathbf{v}(\mathrm{k})=\mathbf{R}^{-1} \mathbf{n}(\mathrm{k})$. The decision for the $\mathrm{n}$ th user is made by $b_{n}(k)=\operatorname{sgn}\left\{\operatorname{Re}\left[z_{n}(k) d_{n}^{*}(k)\right]\right\}$. The performance is affected by the enhanced noise $\mathbf{v}(\mathrm{k})$.

The two-stage detector (2S) considered here uses the decorrelator as the first stage [2]. The tentative decisions from the decorrelator are used to reconstruct the MAI in the second stage. The reconstructed MAI of the n-th user,

$M A I_{n}(k)=\sum_{i=1, i \neq n}^{N} \mathbf{R}_{n, i} \mathbf{W}_{i, i}(k) B_{i}(k)\left(\mathbf{W}_{i, i}(k)=\sqrt{E b_{i}} \mathcal{C}_{i}(k)\right)$, is then subtracted from the output of the matched filter bank (13). 
The final decision for the $\mathrm{n}$-th user is made after canceling its interference, i.e., $\boldsymbol{b}_{n}(k)=\operatorname{sgn}\left\{\operatorname{Re}\left[\left\{y_{n}(k)-M A I_{n}(k)\right\} C_{n}^{*}(k)\right]\right\}$.

The decision-feedback detector (DF) [3] arranges the outputs of the matched filter on the decreasing order of the users' strengths. The reordered cross-correlation matrix $\mathbf{R}^{\mathrm{s}}(\mathrm{k})$ is factored into $\mathbf{R}^{\mathrm{s}}(\mathrm{k})=\mathbf{F}^{\mathrm{s}}(\mathrm{k})^{\mathrm{T}} \mathbf{F}^{\mathrm{s}}(\mathrm{k})$ by Cholesky factorization, where $\mathbf{F}^{\mathrm{s}}(\mathrm{k})$ is a left lower triangular matrix. Applying the noise-whitening filter $\left(\mathbf{F}^{\mathrm{s}}(\mathrm{k})^{\mathrm{T}}\right)^{-1}$ to the sorted output of the matched filter bank gives a new output vector:

$$
\mathbf{f}^{s}(k)=\mathbf{F}^{s}(k) \mathbf{W}^{s}(k) \mathbf{b}^{s}(k)+\mathbf{f}^{s}(k) .
$$

The demodulation of each user is done in a successive fashion. The decision of the $\mathrm{n}$-th strongest user can be expressed by

$$
b_{n}^{k}(k)=\operatorname{sgn}\left\{\operatorname{Re}\left[\left\{\tilde{y}_{n}^{s}(k)-\sum_{m=1}^{n-1}\left[\mathbf{F}^{s}(k)\right]_{n, m}\left[\mathbf{W}^{s}(k)\right]_{m, m} b_{m}^{k}(k)\right\} C_{n}^{s^{*}}(k)\right]\right\} \text {. }
$$

The basic concept of the successive interference cancellation scheme (SIC) is similar to the DF; it demodulates the users sucessively according to their strength order. The algorithm of the SIC is described as follows:

step 1: Sort updated matched filter outputs of $M$ users that were not demodulated previously based on their magnitudes such that $\left|y_{1}^{s}(k)\right| \geq\left|y_{2}^{s}(k)\right| \geq \ldots \geq\left|y_{M}^{s}(k)\right|$. (In the first iteration, $\mathrm{M}=\mathrm{N}$, and actual matched filter outputs are used, $\left.y_{i}^{s}(k)=y_{i}(k), \mathrm{i}=1, \ldots, \mathrm{N}\right)$

step 2: Make a decision for the strongest user among $\mathrm{M}$ remaining sorted users using $y_{1}^{s}(k)$

step 3: Reconstruct the MAI for other remaining users due to the strongest user, i.e., $M A I_{i}=\mathbf{R}_{i, 1}^{s} y_{1}^{s}(k)(\mathrm{i}=2, \ldots \mathrm{M})$.

step 4: Update the matched filter outputs by subtracting the

MAI, i.e., $y_{i}^{s}(k) \leftarrow y_{i}^{s}(k)-M A I_{i}(k)(\mathrm{i}=2, \ldots, \mathrm{M})$. Set

$M \leftarrow M-1$.

Repeat the loop until every user is demodulated.

Note that our SIC detector is different from the detector in [4] for BPSK. In [4], the magnitudes of the soft decisions instead of matched filter outputs are used in sorting (step 1) and in reconstructing the MAI (step 3). Therefore, the phase estimates are needed. On the other hand, our SIC for DPSK can operate without the channel estimates if the information bit is demodulated noncoherently.

The PIC considered here is identical to the approximate decorrelator [5]. The PIC directly uses the outputs of the matched filter bank to reconstruct the MAI for all users in a parallel fashion and therefore does not require ordering of users as in the DF and the SIC. With the definition $\mathbf{R}=\mathbf{I}+\mathbf{L}$ (I is $\mathrm{NxN}$ identity matrix), the signals after MAI cancellation in the $\mathrm{PIC}$ is in a more compact form [17]

$$
\mathbf{f}(k)=\mathbf{R} \mathbf{y}(k)=\mathbf{R} \mathbf{W}(k) \mathbf{b}(k)+\mathbf{n}(k),
$$

where $\mathbf{R}=\mathbf{I}-\mathbf{L}, \mathbf{R}=\mathbf{R} \mathbf{R}$, and $\mathbf{n}(k)=\mathbf{R} \mathbf{n}(k)$. The operation is linear and it is in fact the first order approximation of $\mathbf{R}^{-1}=\mathbf{I}-\mathbf{L}+\mathbf{L}^{2}-\ldots$, therefore it is called the approximate decorrelator in [5].

Below we summarize several issues which arise when the multiuser detectors are operated in conjunction with the Kalman channel estimators [13].

1. Reception of differentially encoded data. As mentioned in Section II, to avoid the burst errors due to the reversal, the data bits should be differentially encoded. The demodulation of differentially encoded data can be coherent--
Differentially Coherent PSK (DC-PSK), or noncoherent-Differential PSK (DPSK) [20]. In the context of multiuser detection, the use of DC-PSK or DPSK depends on the structure of the multiuser detector. If the multiuser detector needs channel estimates in order to regenerate and cancel the MAI (e.g., the $2 \mathrm{~S}$ and the DF), DC-PSK has to used to make the use of the channel estimates. If the MAI reconstruction requires no channel estimate (e.g. the decorrelator, the SIC and the PIC), DPSK can be used to avoid channel estimation. Of course, when channel estimate is feasible, the latter detectors can also use DC-PSK.

2. Disjoint channel estimation. The output of the decorrelator for the $\mathrm{n}$-th user is used as the observation process which is the input to the Kalman filter for this user [15]. There are $\mathrm{N}$ channel estimators for $\mathrm{N}$ users. The purposes of disjoint estimation are to reduce the complexity (dimension) of the Kalman filter, and to remove the data dependency in the error covariance matrix so that the error covariance matrix can be computed off-line [13, 19].

\section{Performance analysis}

In $[12,13]$, the closed form expressions for the BERs of the decorrelator, the $2 \mathrm{~S}$ and the DF with the Kalman channel estimators were derived. The derivation is based on the fact that the Kalman filter is the MMSE estimator when the fading channel obeys the Gauss-Markov model (e.g. an AR process), and the signal model of the Kalman filter is identical to the fading channel. In this paper, since we use the modified Jakes fading channel model, the signal model of the Kalman filter is not identical to the channel model, and thus the Kalman filter is not the MMSE estimator any more. Therefore, the closed form expression for the BERs cannot be derived; the BERs of these detectors with the Kalman channel estimator are evaluated using simulation.

For multiuser detectors with DPSK, the BER can be analyzed using the unified analysis $[11,12]$. The BER of the decorrelator with DPSK is [11]

$$
P E_{d e c, D P S K}=\frac{1}{2}\left[1-\frac{\Phi(1)}{1+\left[\mathbf{R}^{-1}\right]_{1,1} / \bar{\gamma}_{1}}\right]
$$

where $\Phi(1)$ is the autocorrelation function (6) evaluated at one sampling period, and $\bar{\gamma}_{1}=E b_{1} E\left\{\left|C_{1}(k)\right|^{2}\right\} / N_{0}=E b_{1} / N_{0}$ is the average SNR of user 1.

The BERs of the conventional detector and the PIC with DPSK can also be calculated using the unified analysis [17]:

$$
\begin{gathered}
P E_{\text {conv }, D P S K}=\frac{1}{2}\left[1-\frac{\Phi(1)}{1+\sum_{j=2}^{N}\left[\mathbf{R}_{1, j}\right]^{2}\left(E b_{j} / E b_{1}\right)+1 / \bar{\gamma}_{1}}\right],(18) \\
P E_{P I C, D P S K}=\frac{1}{2}\left[1-\frac{\Phi(1)}{1+\sum_{j=2}^{N}\left(\mathbf{R}_{1, j} / \mathbf{R}_{1,1}\right)^{2}\left(E b_{j} / E b_{1}\right)+\left(\rho /\left[\mathbf{R}_{1,1}\right]^{2}\right)\left(1 / \bar{\gamma}_{1}\right)}\right]
\end{gathered}
$$

where $\rho=\left[\mathbf{R} \mathbf{R} \mathbf{R}^{T}\right]_{1,1}$, the enhanced additive noise power by the linear operation $\mathbf{R}$ on the output of matched filter. The BER of the SIC with DPSK is evaluated by simulation since the analysis is not obvious due to the sorting process.

From (17-19), the BERs of all these detectors with DPSK depend on the fading rate, which is quantified by $\Phi(1)$. The performance of the conventional detector and the PIC also 
depends on the signal strengths of the interferers as well as the strength of the desired users, whereas the decorrelator only depends on the strength of the desired user. Therefore, the decorrelator is near-far resistant. These three detectors have error floors as $\bar{\gamma}_{1} \rightarrow \infty$. The error floor of the decorrelator is determined only by the fading rate and agrees with the error floor for the single user with DPSK. However, the error floors of the PIC and the conventional detector are greater since they also depend on the cross-correlation values and the strengths of other users.

\section{Numerical examples}

The channel coefficient is generated from the modified Jakes model with 24 distinct oscillators, or 96 oscillators in total. The Maximum Doppler frequency is $80 \mathrm{~Hz}$, which corresponds to the vehicle speed of $60 \mathrm{mph}$ and the carrier frequency of $900 \mathrm{MHz}$. The sampling rate is $10 \mathrm{Kbps}$. By using the Walsh-Hadamard codewords, we generate 24 uncorrelated fading waveforms [9]. The parameters in the Kalman filter are chosen based on the approximate second order AR signal model of the modified Jakes model, as discussed in Section II. The data bits are differentially encoded to avoid burst errors due to reversal.

We simulate the scenarios for 2,4 , and 24 users, as shown in Figures 2-4. All users have the same average SNR. The normalized signature cross-correlation for 2 users is 0.9 . The signature sequences of 4 users and 24 users are chosen from Gold sequences of length 7 and 31 respectively. Their normalized signature cross-correlation matrices $\mathbf{R}$ are the same as in $[13,24]$. The performance of the $2 \mathrm{~S}$ and the DF is very close and we only present the result of the $2 \mathrm{~S}$ for clarity.

The simulation results are similar to the results of [13] where the fading channel was modeled as a second order AR process. When coherent reception (DC-PSK) is used, the performance gain of the $2 \mathrm{~S}$ and the DF over the decorrelator is observed only for low SNR values, and is not as significant as in the case of perfect channel estimation [21, 22]. For high SNR values, these three detectors have comparable performance. This observation results from the same fact as in [13] -- the estimation error is irreducible and becomes the dominant factor in the high SNR region. Note that the error floors of these detectors under channel mismatch are not obvious, but their existence can clearly be shown if the fading rate is higher [17].

For noncoherent reception (DPSK), the SIC and the PIC have higher error floors than the decorrelator for more than two users. For the two-user case, since there is only one interferer, the interference can be canceled more completely compared with the case of more users. The two-user scenario is also a special case for the PIC; its performance is identical to that of the decorrelator [5]. For more than two users, the accumulation of the residual interference after MAI cancellation limits the performance of the SIC and the PIC. Therefore, they have much higher error floors.

The decorrelator with noncoherent reception reaches the same error floor regardless of the number of users. The limiting performance of the decorrelator is only determined by the fading rate as discussed in Section IV.

In summary, in our comparison, the decorrelator seems to be the most robust scheme in presence of channel mismatch. In addition, it has moderate complexity and is easier to implement than the $2 \mathrm{~S}$ and the DF.

\section{Conclusions and Future work}

We have shown that the Kalman filter based on a second order AR signal model can be used to estimate a realistic flat Raleigh fading channel. The data bits are encoded differentially to avoid the long burst errors due to the reversal. The closed form expression for the BERs of the conventional detector and the PIC with DPSK are derived using the unified analysis. The performance of the multiuser detectors with the Kalman channel estimators-the $2 \mathrm{~S}$, the DF, and the decorrelator is assessed by simulation. The result shows that the decorrelator, with the lowest complexity, is the most robust under channel mismatch. Several noncoherent multiuser detectors which do not require channel estimation-the conventional detector, the PIC, the SIC, and the decorrelator, are also compared. The performance of the PIC and the SIC degrades as number of users increases whereas the decorrelator is only affected by the fading rate. The result confirms the robustness of the decorrelator. For lower SNR, the performance of the decorrelator with or without the channel estimators is almost same. Therefore, the decorrelator is a more desirable multiuser detector for practical use. Future work includes channel estimation, performance comparison of multiuser detectors for mismatched multipath fading channels, as well as practical implementation of the decorrelator.

\section{Support}

This work was supported by the IBM Fellowship, the Center for Advanced Computing \& Communication at North Carolina State University and NSF Grant NCR-9410227.

\section{Reference}

[1] R. Lupas, S. Verdu, "Linear Multiuser Detectors for Synchronous Code-Division Multiple-Access Channel," IEEE Transactions on Information Theory, Vol. IT-35, No. 1, Jan. 1989, pp. 123-136.

[2] M.K. Varanasi, B. Aazhang, "Near-optimum Detection in Synchronous Code-Division Multiple Access Channel," IEEE Transactions on Communications, Vol. COM-39, No. 5, May 1991, pp. 725-736.

[3] A. Duel-Hallen, "Decorrelating Decision-Feedback Multiuser Detector for Synchronous Code-Division Multiple Access Channel," IEEE Transactions on Communications, Vol. COM-41, No. 2, Feb. 1993, pp. 285-290.

[4] P. Patel, J. Holtzman, "Performance Comparison of a DS/CDMA system using a Successive Interference cancellation (IC) scheme and a Parallel IC Scheme under Fading," ICC 94, pp. 510-515, New Orleans, LA, 1994.

[5] N.B. Mandayam, S. Verdu, "Analysis of an Approximate Decorrelating Detector," Proc. of Thirty-Third Annual Allerton Conference on Communication, Control and Computing, Monticello, Oct. 1995, pp. 1043-1052.

[6] P.Y. Kam, "Optimal Detection of Digital Data over the Nonselective Rayleigh Fading Channel with Diversity Reception," IEEE Transactions on Communications, Vol. COM-39, No. 2, Feb. 1991, pp. 214-219.

[7] R. Haeb, H. Meyr, "A Systematic Approach to Carrier Recovery and Detection of Digitally Phase Modulated Signals on Fading Channels," IEEE Transactions on Communications, Vol. 37, No. 7, July 1989, pp. 748754. 
[8] W.C. Jakes, Jr., Microwave Mobile Communication, John Wiley \& Sons, New York, 1974, p. 75.

[9] P. Dent, G.E. Bottomley, T. Croft, "Jakes Fading Model Revisited," IEEE Electronics Letters, Vol. 29, No. 13, June 1993, pp. 1162-1163.

[10] S. Vasudevan and M.K. Varanasi, "Achieving NearOptimum Asymptotic Efficiency and Fading Resistance over the Time-varying Rayleigh-faded CDMA Channel," to appear in IEEE Transactions on Communications, Vol. COM-44, No. 9, Sept. 1996.

[11] Z. Zvonar, Multiuser Detection for Rayleigh Fading Channels, Ph.D. Thesis, Chapter 4, Northeastern University, Boston, Massachusetts, 1993.

[12] Z. Zvonar, M. Stojanovic, "Performance of Multiuser Diversity Reception in Nonselective Rayleigh Fading CDMA Channels," Proc. of the Third Communication Theory Mini-Conference (CTMC '94), San Francisco, CA, Nov. 1994, pp. 171-175.

[13] H.Y. Wu, A. Duel-Hallen, "Multiuser Detection with Differentially Encoded Data for Mismatched Flat Rayleigh Fading CDMA Channels," to appear in Proc. of the 30th Annual Conference on Information Sciences and Systems, Princeton University, Princeton, NJ, March 1996.

[14] H. Brehm, W. Stammler, M. Werner, "Design of a Highly Flexible Digital Simulator for Narrowband Fading Channels," EUSIPCO-86 Proce. Signal Processing III: Theories and Applications, I. T. Young, Ed. Elsevier Science Publishes B.V. (North Holland). pp. 1113-1116, 1986.

[15] L. Lindbom, "Simplified Kalman Estimation of Fading Mobile Radio Channels: High Performance at LMS Computational Load," IEEE ICASSP, Vol. 3, April 1993, pp. 352-355.

[16] L. Lindbom, A Wiener Filtering Approach to the Design of Tracking Algorithms with Applications in Mobile Radio Communications, Ph.D. Thesis, Uppsala University, Uppsala, Sweden, 1995.

[17] H.Y. Wu, Multiuser Detection and Channel Estimation for Synchronous CDMA Channels, Ph.D. Thesis, North Carolina State University, Raleigh, North Carolina, in preparation.

[18] B.D.O. Anderson and J.R. Moore, Optimal Filtering, Prentice-Hall, 1979.

[19] H.Y. Wu, A. Duel-Hallen, "Channel Estimation and Multiuser Detection for Frequency-Nonselective Fading Synchronous CDMA Channel," Proc. of Thirty-Second Annual Allerton Conference on Communication, Control and Computing, Monticello, Sept. 1994, pp. 335-344.

[20] J. G. Proakis, "Digital Communication," McGraw-Hill, 3rd edition, 1995.

[21] H.Y. Wu, A. Duel-Hallen, "Performance Comparison of Multiuser Detectors with Channel Estimation for Flat Rayleigh Fading CDMA Channels," Submitted to Special Issue on "Interference in Mobile Wireless Systems, " Journal of Wireless Personal Communications, Kluwer, to appear in July 1996.

[22] H.Y. Wu, A. Duel-Hallen, "Performance of Multiuser Decision-Feedback Detectors for Flat Fading Synchronous CDMA Channels," Proc. of the 28th Annual Conference on Information Sciences and
Systems, Princeton University, Princeton, NJ, March 1994, pp. 133-138.

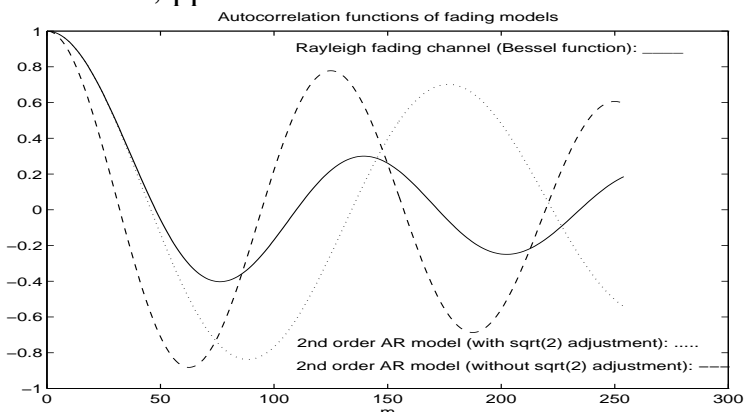

Figure 1: Autocorrelation functions of fading channel models

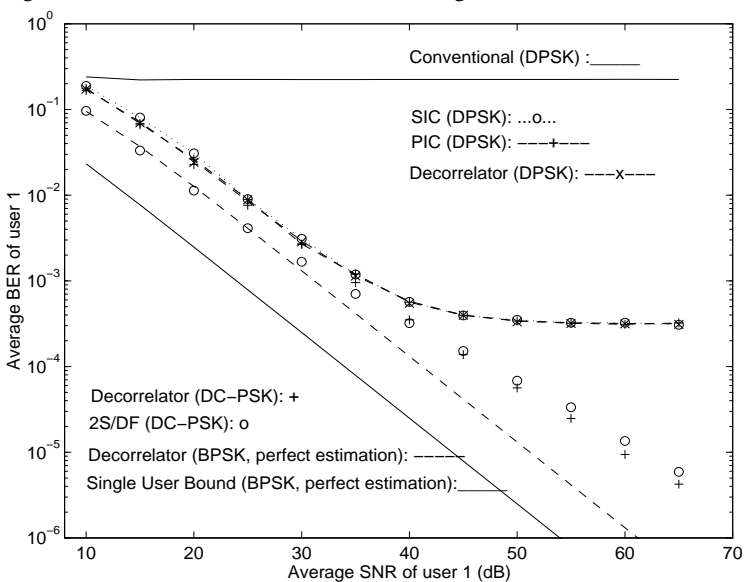

Figure 2: Performance comparison for a 2-user channel with mismatch

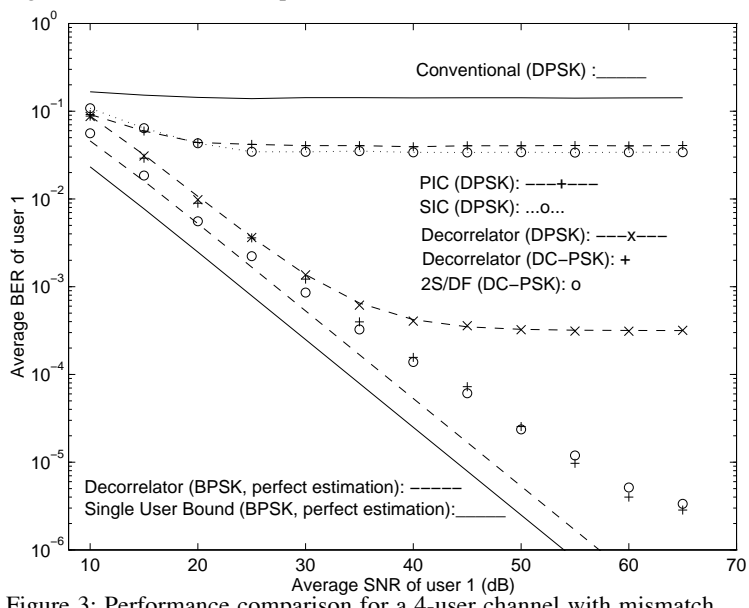

Figure 3: Performance comparison for a 4-user channel with mismatch

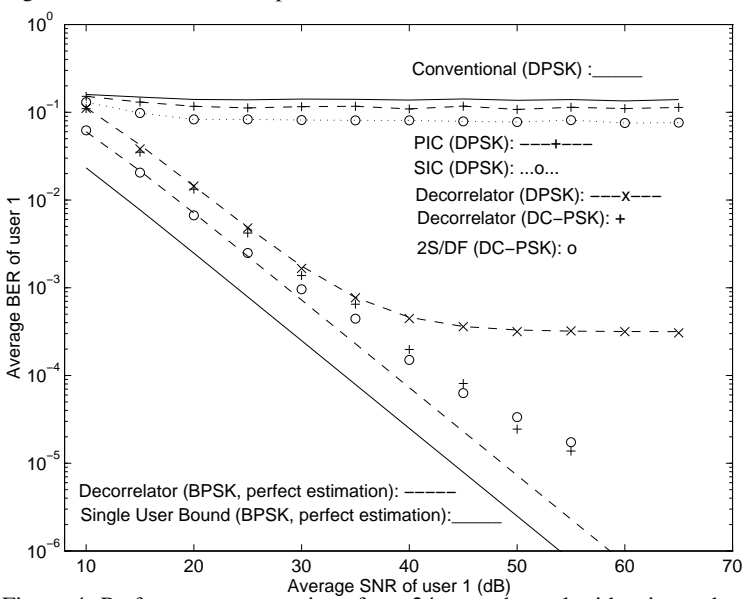

Figure 4: Performance comparison for a 24 -user channel with mismatch 